\title{
How could static telepathology improve diagnosis in neuropathology?
}

\author{
Myriam Remmelinck $^{\mathrm{a}}$, M. Beatriz S. Lopes ${ }^{\mathrm{b}}$, \\ Nathalie Nagy ${ }^{\mathrm{a}}$, Sandrine Rorive ${ }^{\mathrm{a}}$, \\ Katja Rombaut ${ }^{\mathrm{a}}$, Christine Decaestecker ${ }^{\mathrm{c}, *}$, \\ Robert Kiss ${ }^{\mathrm{c}, * *}$ and Isabelle Salmon ${ }^{\mathrm{a}, * * *}$ \\ ${ }^{a}$ Department of Pathology, Cliniques Universitaires \\ de Bruxelles, Hôpital Erasme, Université Libre de \\ Bruxelles, Brussels, Belgium \\ ${ }^{\mathrm{b}}$ Division of Neuropathology, Department of \\ Pathology, University of Virginia Health Sciences \\ Center, Charlottesville, VA, USA \\ ${ }^{\mathrm{c}}$ Laboratory of Histopathology, Faculty of Medicine, \\ Université Libre de Bruxelles, Brussels, Belgium
}

The present paper reports our experience with, and our opinion of static telepathology as applied to neuropathology by means of the PHAROS acquisition system and conventional telephone data transmission (modem). The classical procedure of expert consultation based on surface mailing of histological slides is routinely performed, especially in highly specialized fields of pathology. Telepathology is an easy means of sharing scientific expertise at international level and could thus improve diagnosis particularly in neuropathology, where certain tumor types are very rare and complex to diagnose. Dynamic telepathology allows the referring pathologist to capture by himself images supporting their diagnosis. Using static telepathology the pathologist could be limited in diagnosis by problems in fields selection.

We devoted a whole year to collecting all the technical parameters characterizing the use of digitized neuropathological data files in order to investigate the feasibility of telepathology and the extent to which its use could improve diagnoses. Our results on a series of 38 histological brain examinations illustrate how we successfully established an international connection between two departments of pathology in Belgium and the USA. The referring pathologists gave

\footnotetext{
*This author is a Research Associate with the "Fonds National de la Recherche Scientifique" (FNRS), Belgium.

${ }^{* *}$ This author is a Senior Research Associate with the "Fonds National de la Recherche Scientifique" (FNRS), Belgium.

**** Corresponding author: Isabelle Salmon, M.D., Department of Pathology, Erasmus Hospital, Free University of Brussels, 808 route de Lennik, 1070 Brussels, Belgium. Tel.: +32 255531 15; Fax: +32 255547 90; E-mail: isalmon@med.ulb.ac.be.
}

\begin{abstract}
diagnoses in 35 cases and deferred only 3 . Despite a timeconsuming procedure for the telepathology session of a few cases, this tool provides easy access to expert diagnosis and real-time discussion, both of which are of considerable interest and offer significant improvements in neuropathology.
\end{abstract}

Keywords: Static telepathology, neuropathology, data transmission, expert consultation

\section{Introduction}

Communication and information, key words in our century, have played striking roles in recent developments in pathology. In this context telepathology has been highly developed over the last 15 years, as indeed, is made clear by Kayser et al. in their well-documented book [11].

In fact, the various fields of activity covered by the terms are now so highly specialized that consensus discussion of difficult cases is required to obtain expert diagnoses. In Erasmus hospital, where neurosurgery is particularly well developed, neuropathology, and especially brain tumor classification, has become a very specialized subfield. In this field, an expert diagnostic panel has emerged as a very important means of increasing the accuracy of clinical diagnosis, especially in the field of pediatric tumors and stereotactic-guided biopsy.

Since 1996 we have tried to replace the surface mailing of histological slides by static telepathology in the expert discussion of difficult cases. With the help of the Division of Neuropathology of the University of Virginia Health Sciences Center, we decided to investigate the extent to which the use of telepathology could improve diagnosis. Furthermore, we devoted a whole year to collect all the technical parameters characterizing the use of neuropathological files: the number and type of images per case, the time needed to set up a complete case file and the time of discussion and transmission. The present paper reports on these data, our experience, and our opinion of static telepathology as applied to neuropathology. 


\section{Materials and methods}

In order to evaluate the real impact of telepathology in routine neuropathological diagnosis we undertook analysis of 38 neuropathological selected surgical cases, raising difficulties in obtaining firm diagnosis, with a view to the different technical parameters of acquisition and transmission of images, and also the ability to perform diagnoses on transmitted images.

\subsection{Technology used}

The PHAROS system (version 1.52 VAMS, Zagreb, Croatia) is the acquisition and transmission software that we used to establish a connection between the Department of Pathology of the Erasmus Hospital of the Free University of Brussels, ULB (Belgium) and the Division of Neuropathology of the University of Virginia (Charlottesville, USA). Both laboratories were equipped with the same PHAROS system. The acquisition station was a 3CCD Sony XC.003P video camera (Japan) connected to an Olympus-BX 50 microscope (Japan) by a universal " $C$ " mount (Olympus). The video camera was connected to a frame grabber (IMS Chroma) in a Pentium PC with 32Mb RAM and $1 \mathrm{~Gb}$ of disk storage. A tape streamer allowed image storage and easy retrieval. Transmissions were affected using Bocamodem (v.34 and $28.800 \mathrm{kbps}$, USA) working in conjunction with conventional telephone networks.

\subsection{Case file design}

Whiteboard is Pharos' basic system for image and text interchange. Whiteboard can contain images, text, and graphical objects such as arrow. The first whiteboard of the transmitted file is related to clinical data including the patient's references, date of birth and sex as well as his/her clinical history, surgical report. Following whiteboards consisted in histological images characterizing the case.

The clinical history consisted of the symptoms reported chronologically, the computed tomography scan and the magnetic resonance imaging features describing in detail the location and the single or multiple appearance of the lesion, the tumor's delineation and the presence/absence of contrast enhancement. No digitized radiological images were transmitted even though the system allows it.

The type of surgery, i.e., stereotactic biopsy, partial biopsy or lobotomy, was reported with a macroscopic description of the surgical specimen when required.
In constituting the set of histological images we decided to follow conventional histological examination procedure: firstly, images from low- to high-power field of H-E $(\times 40, \times 100, \times 200, \times 400)$ stained slides were transmitted and secondly, if necessary for the diagnoses, special staining and immunohistochemistry images. Low-power field H-E images were taken in order to be able to appreciate the general appearance of the lesion (e.g., the surgical margins or the presence of normal tissue around the lesion) with intermediatepower ones to describe the different appearance of the histological architecture and high-power ones to evaluate cytological features such as nuclear pleomorphism, cytoplasm content, mitosis and so on. With respect to special staining and immunohistochemistry, annotations concerning the type of antibody or special staining were appended to each file. We used dynamic arrows on the image to specify special immunohistological or histological features. Images of previous surgical examinations were included if necessary.

\subsection{Communication procedure}

Time schedule was defined for sending selected images from the local to the referring pathologist. The discussion was made immediately after images transmission, for a few cases, after preliminary discussion, a second transfer of different histological area was made to obtain a final diagnosis. The PHAROS system allowed two way of communication, first by sharing whiteboard and secondly by using text-only chat option. For discussion we have used the chat window. Images were stored after the session.

\section{Results}

\subsection{Clinical data}

The majority of the cases (34 out of 38 ) consisted of brain tumors in patients aged from 10 days to 65 years. About $50 \%$ of the patients were younger than 16. The 4 non-neoplastic lesions appeared in the context of infectious diseases, principally in immunocompromised patients. Five of the 38 cases were stereotactic examinations and 33 were obtained through conventional surgery. No frozen section images or macroscopic ones were transmitted. 


\subsection{Set-up of data file}

Between 5 and 15 images were transmitted for each case. As we were able to include a maximum of $3 \mathrm{im}$ ages per whiteboard, each complete data file had between 3 and 9 whiteboards, depending on case heterogeneity. While only H-E images were transmitted for 13 of the cases, immunohistostaining images were also provided for 25. Cytological smear images were added in 7 cases.

From a technical point of view, the PHAROS system was very easy to use, with a quick set-up procedure enabling the files to be acquired (and transmitted, as explained in the following section). All these stages were passed through without any major technical problems.

We faced slight problems in image acquisition only:

- Our system used a universal "C" mount cannot be equipped with an ultra-wide field for optical reasons, and so allowed only $70 \%$ of the microscope field to be covered by the camera. This caused a discrepancy between the microscope field and the image recorded.

- An absence of perfect focus adjustment between the nose piece of the microscope and the camera caused certain degree of annoyance.

- Our version of the PHAROS system had only a limited capacity for inserting clinical data due to an insufficient word-processing system.

- The time required to set-up a complete file appeared to be slightly time-consuming, varying as it did from 15 to 30 minutes according to the number of images and the amount of clinical data.

\subsection{File transmission}

The communication procedure was well designed and very prompt for application.

- Using conventional telephone networks, Bocamodem was a source of very efficient transmission. In 1 year, only 2 interruptions obliged us to restart transmission. The speed of the transmissions varied with the number and type of images sent, with the transmission of each whiteboard taking from 90 to 360 seconds (with an average of 150). In average, a complete data file was transmitted in 15 minutes.

\subsection{Interactive expert diagnostic procedure and diagnoses performed}

The diagnostic procedure consisted of real-time discussion on the basis of images seen simultaneously by both teams of pathologists. After the individual examination of the different images the discussion, using a chat system, last from 5 to 15 minutes (mean $=8 \mathrm{~min}$ ). During the discussion interactively usable indicators projected on the images were used to underline specific histological features.

Table 1 illustrates the consensus diagnosis obtained in 35 cases. For the remaining 3 cases, glass slides had to be mailed. The first deferred case was a vasculitic lesion with a differential diagnosis of lymphoma; the second was a stereotactic specimen from a brain tumor, and the third was a pediatric tumor causing grading difficulties. The diagnoses obtained after telepathology discussion (35 cases) or slides review (3 cases) were carried out according to the WHO Histological Classification of Nervous System Tumors [13]. They included 19 glial tumors consisting of 8 astrocytomas (3 grade I, 3 grade II and 2 grade III tumors), 6 oligodendrogliomas ( 2 grade II and 4 grade III tumors) and 5 mixed oligo-astrocytomas. We also succeeded in classifying 5 PNET tumors, 1 meningioma, 5 soft tissue lesions, 1 lymphoma, 3 germ cell tumors and 4 non-tumor lesions.

\section{Discussion}

Neuropathology, and particularly brain tumor diagnosis, is becoming more and more specialized because of the complexity of histological classifications and the use of different markers originating from immunohistochemistry and molecular genetics. Only a few pathologists in any country are experienced in this area, and requests for consultations are thus usual practice in this field. Telepathology is an easy way of sharing scientific expertise at international level and could thus improve diagnosis particularly in neuropathology, where certain tumor types are very rare and complex to diagnose.

Developments in videotechnology, high performance PCs and progress in data transmission enable pathological diagnoses to be performed on high quality digitized images.

Our results illustrate how we successfully established an international connection between two departments of pathology, one in Belgium and one in 
Table 1

Diagnoses obtained using telepathology panel discussion

\begin{tabular}{lcl}
\hline Tumor type & $\begin{array}{c}\text { Number of cases } \\
(38 \text { cases })\end{array}$ & Discordance \\
\hline Glial tumors & & \\
Astrocytomas & 3 & GI/GII \\
Astrocytomas grade I & 3 & GII/GIII \\
Astrocytomas grade II & 2 & \\
Astrocytomas grade III & & \\
Oligodendrogliomas & 2 & \\
Oligodendrogliomas grade II & 4 & \\
Oligodendrogliomas grade III & 5 & \\
Mixed oligo-astrocytomas & 5 & \\
PNET (primitive neuroectodermal tumors) & 1 & \\
Meningeal tumors & 5 & \\
Sarcomas & 1 & \\
Lymphomas & 3 & \\
Germ cell tumors & 4 & \\
Non-tumoral diseases & & \\
& &
\end{tabular}

the USA. As demonstrated by numerous studies (see, for example, [5-7,16]), these intercontinental consultations are now carried out and more commonly use the Internet. As published in [2], dynamic telepathology is also used successfully to perform primary diagnoses in routine surgical pathology.

In his book Kayser details all the countries now involved in telepathology and all the different applications developed [11]. Telepathology has been used not only for obtaining primary diagnoses and the expert referral of difficult cases, or consensus diagnosis for pathological reviews, but has also been used for teaching students and setting up data banks of pathological cases. In addition Sowter and Wells underline the importance of telepathology in quality assurance programs [17].

Intra-operative diagnosis is now considered as one of the more interesting applications of telepathology: as it has been shown by the numerous studies published on static and dynamic telepathology applied to remote frozen section diagnosis $[10,14]$.

Expert consultation on pathological cases has been successfully developed between several universities in the United States and Europe for a number of years (see a review in [12]). As mentioned above, diagnosis based on expert consensus is our principal interest in the routine use of telepathology. With our system we succeeded in carrying out 35/38 consensus diagnoses of difficult neurosurgical cases. Only a small number of studies report other experiences in telepathol- ogy specifically applied to neuropathology. One study shows the effectiveness of telepathology in assisting the diagnosis of 52 neurosurgical frozen section cases for which only 7 were the subject of major discrepancies [3]. Another reports an application to the intraoperative examination of stereotactic brain tissue successfully carried out by the Neurosurgery Department of Mannheim University [1].

In contrast to the infrequent use of telepathology in neuropathology, many studies on telepathology concern routine surgical pathology. As stated by Wells and Sowter [18] current statistics are that $75 \%$ of cases can be diagnosed effectively on digitized images without the need to send slides for analysis. Similarly to Halliday et al. [8], we report the feasibility and the diagnostic accuracy of international static image telepathology. The series of Halliday et al. consisted of cases from different areas of pathology, such as gastrointestinal, gynecological, lymph node, bone and sarcomas for the most part (including 8 neuropathological cases) [8]. In their study, telepathologists were able to perform 127 diagnoses and deferred only 27 cases (non-neuropathological ones). These authors [8] critically examined the effectiveness of telepathology by analyzing the concordance between telepathologybased and glass slide-based diagnoses; this concordance was around $88 \%$. As in our personal experience, the authors noted that the major limitations of static telepathology are inappropriate field selection and sampling biases on the part of the referring pathol- 
ogists. Another study also reports $87.5 \%$ diagnostic concordance between CD-ROM digitized images and conventional glass sides; the discordances observed were mostly related to image selection and/or quality [19]. In fact, both the above studies and others emphasize the importance in the selection of the histological fields and the increase of diagnostic accuracy when images are selected by expert pathologists [4, 20]. These problems were not so significant in our experience probably because both pathologists had the opportunity to work together in Charlottesville before the telepathology sessions, and thus used the same field selection procedure and attached similar importance to each diagnostic criterion. Dynamic telepathology avoid selection bias by enabling the interpreting pathologists to select histological fields themselves, in the same way as using a conventional microscope [2].

Other common sources of diagnostic error, due to deficiencies in image quality were not encountered in our study. The slight technical problems connected with image acquisition reported under Results could be easily resolved by means of an ultra-wide field $\mathrm{C}$ mount.

Our opinion is that the only serious problem with telepathology concerns the time required for the complete procedure (file set-up, transmission and discussion), which may appear forbidding. However, as reported by Kayser [10], the classical consultative procedure in pathology, as it is based on the mailing of slides, is in fact also expensive and time-consuming. As shown under Results, the time taken for a complete telepathology session is at least 25 min, with a mean time of $40 \mathrm{~min}$. Similar data are reported by Eusebi et al. [7], who used static telepathology between the University of Bologna (Italy) and the Memorial SloanKettering Cancer Center (USA).

In practice, our procedure for reducing the time required for a telepathology session consisted of first transmitting the complete file (including all the images and clinical data). The real-time discussion was in fact done later to enable the referring pathologist to have all the time required for the case analysis. If he/she needed more information, new images could be send before the real time discussion. However, this second data transmission increased the time required for the complete procedure. We thus believe that it is important to set up a very complete data file directly, before discussion.

ISDN transmission is able to reduce the time necessary for telepathology consultation, as, proved by different series in the literature. For instance, one of the first studies on ISDN transmission published in 1993 reports that the time required for a diagnostic session in static telepathology was between 25 and 35 min [15]. In transplantation pathology, Ito et al. developed static telepathology with ISDN transmission, so enabling short sessions of on average 13 min [9].

In conclusion, the static telepathology system that we use provides us with accurate help in neuropathological diagnosis. The procedure that we have developed is easy to use, provides high-quality digitized images and complete pathological files, including clinical data, which can also be used for training. One slight problem encountered is its time-consuming nature, but the system has the advantage of being cheaper than dynamic telepathology. Telepathology provides easy access to expert diagnosis and real-time discussion, both of which are of considerable interest and offer significant improvements in neuropathology.

\section{References}

[1] H.R. Abbasi, R. Weigel, C. Sommer, P. Schmiedek and M. Kiessling, Telepathology in neurosurgery, Stud. Health. Technol. Inform. 62 (1999), 1-7.

[2] U.A. Almagro, B.E. Dunn, H. Choi and D.L. Recla, Telepathology (letter to the editor), Am. J. Surg. Pathol. 22 (1998), 11611163.

[3] R.L. Becker, Jr., C.S. Specht, R. Jones, M.E. Rueda Pedraza and T.J. O'Leary, Use of remote video microscopy (telepathology) as an adjunct to neurosurgical frozen section consultation, Hum. Pathol. 24 (1993), 909-911.

[4] P.W. Callas, K.O. Leslie, A.R. Mattia, D.L. Weaver, D. Cook, B. Travis, D.E. Stanley, L.A. Rogers, S.L. Mount, T.D. Trainer, M.A. Zarka and R.M. Belding, Diagnostic accuracy of a rural live video telepathology system, Am. J. Surg. Pathol. 21 (1997), 812-819.

[5] V. Della Mea, F. Puglisi, M. Bozanini, S. Forti, V. Amoroso, R. Visentin, P. Dalla Palma and C.A. Beltrami, Fine needle aspiration cytology of the breast: a preliminary report on telepathology through Internet multimedia electronic mail, Mod. Pathol. 10 (1998), 636-641.

[6] M. Eusebi, L. Foschini, S. Grae and J. Rosai, Transcontinental consults in surgical pathology via the Internet, Hum. Pathol. 28 (1997), 13-16.

[7] M. Eusebi, L. Losi, S. Erde and J. Rosai, Telepathology: a powerful tool for improved communication among pathologists, Curr. Diagn. Pathol. 4 (1997), 73-75.

[8] B.E. Halliday, A.K. Bhattacharyya, A.R. Graham, J.R. Davis, S.A. Leavitt, R.B. Nagle, W.J. Mc Laughlin, R.A. Rivas, R. Martinez, E.A. Krupinski and P.S. Weinstein, Diagnostic accuracy of an international static-imaging telepathology consultation service, Hum. Pathol. 28 (1997), 17-21.

[9] H. Ito, H. Adachi, K. Taniyama, Y. Fukuda and K. Dohi, Telepathology is available for transplantation pathology, experience in Japan using an integrated, low cost, and high quality system, Mod. Pathol. 7 (1994), 801-805. 
[10] K. Kayser, P. Fritz, M. Drlicek and W. Rahn, Expert consultation by use of telepathology: the Heidelberg experiences, Anal. Cell. Pathol. 9 (1995), 53-60.

[11] K. Kayser, J. Szymas and R. Weinstein, Telepathology, Telecommunication, Electronic Education and Publication in Pathology, Springer, Berlin, 1999.

[12] K. Kayser and G. Kayser, Basic aspect of and recent developments in telepathology in Europe, with specific emphasis on quality assurance, Anal. Quant. Cytol. Histol. 21 (1999), 319328.

[13] P. Kleihues, B.C. Burger and B.W. Scheithauer, Histological Typing of Tumours of the Central Nervous System, 2nd edn, Springer-Verlag, Berlin, 1993.

[14] I. Nordrum, B. Engum, E. Rinde, A. Finseth, H. Ericsson, M. Kearney, H. Stalsberg and T.J. Eide, Remote frozen section service, a telepathology project in northern Norway, Hum. Pathol. 22 (1991), 514-518.

[15] M. Oberholzer, H.R. Fischer, H. Christen, S. Gerber, M. Bruehlmann, M. Mihatsch, M. Famos, C. Winkler, P. Fehr, L. Bächtold and K. Kayser, Telepatholgogy with ISDN - a new tool for image transfer in surgical pathology, Hum. Pathol. 24 (1993), 1078-1085.

[16] I. Petersen, G. Wolf, K. Roth and K. Schlüns, Telepathology by the Internet, J. Pathol. 191 (2000), 8-14.

[17] C. Sowter and C.A. Wells, System requirements, the use of telepathology in diagnostic pathology and its application to quality assurance programs, Curr. Diagn. Pathol. 4 (1997), 6572.

[18] C.A. Wells and C. Sowter, Telepathology: a diagnostic tool for the millennium?, J. Pathol. 191 (2000), 1-7.

[19] D.S. Weinberg, F.A. Allaert, P. Dusserre, F. Drouot, B. Retailliau, W.R. Welch, J. Longtine, G. Brodsky, R. Folkerth and M. Doolittle, Telepathology diagnosis by means of digital still images, an international validation study, Hum. Pathol. 27 (1996), 111-118.

[20] M.H. Weinstein and J.I. Epstein, Telepathology diagnosis of prostate needle biopsies, Hum. Pathol. 28 (1997), 22-29. 


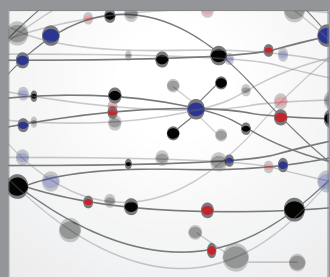

The Scientific World Journal
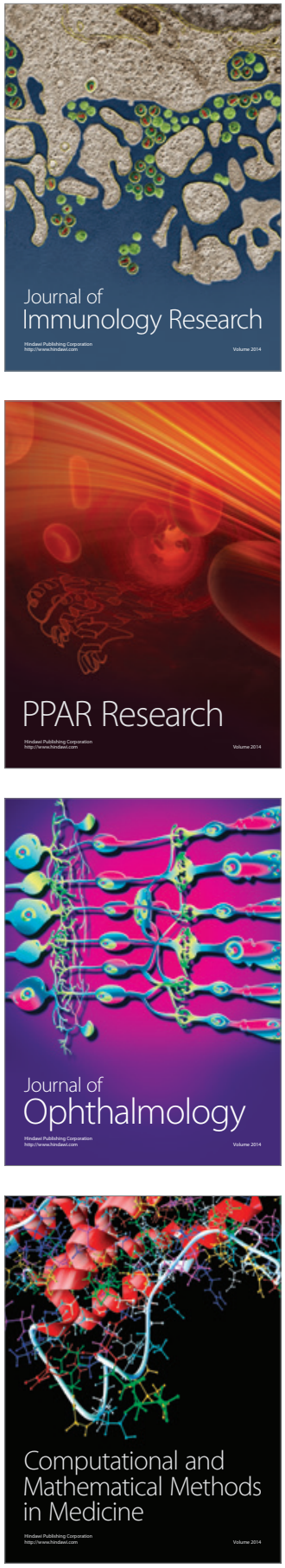

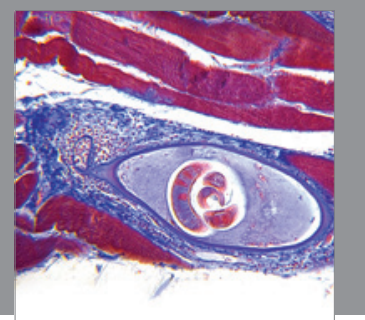

Gastroenterology

Research and Practice
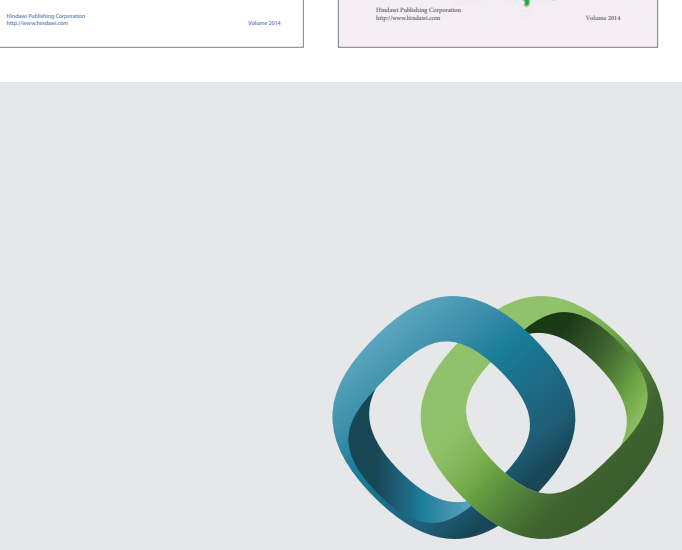

\section{Hindawi}

Submit your manuscripts at

http://www.hindawi.com
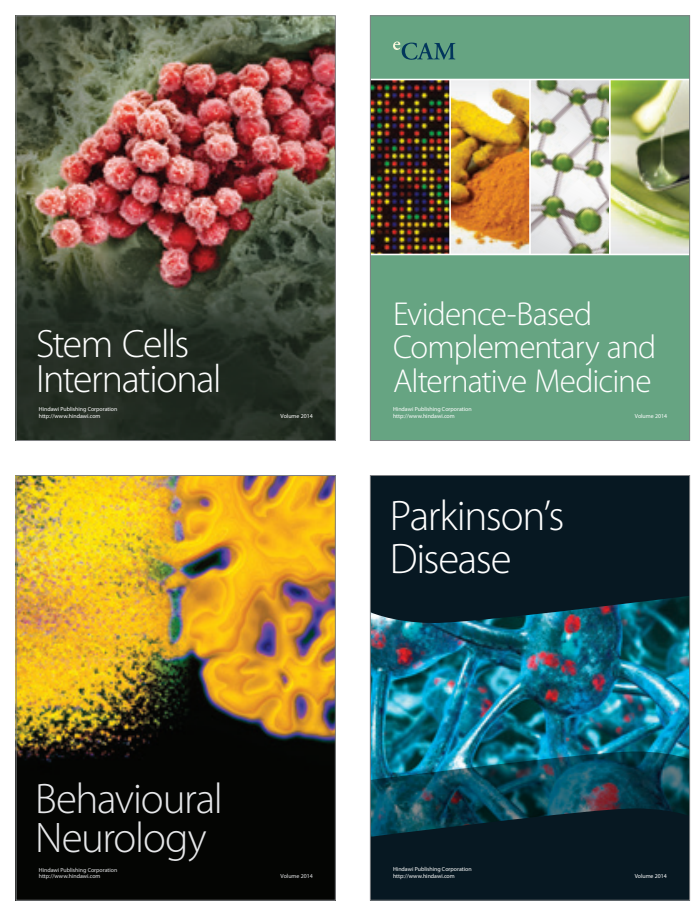

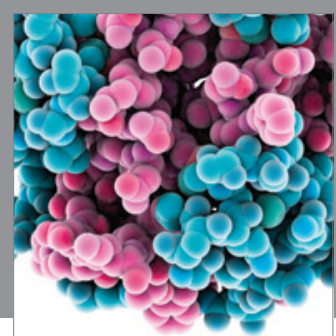

Journal of
Diabetes Research

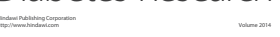

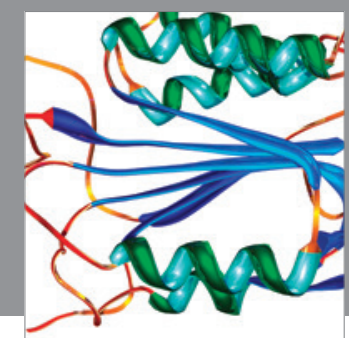

Disease Markers
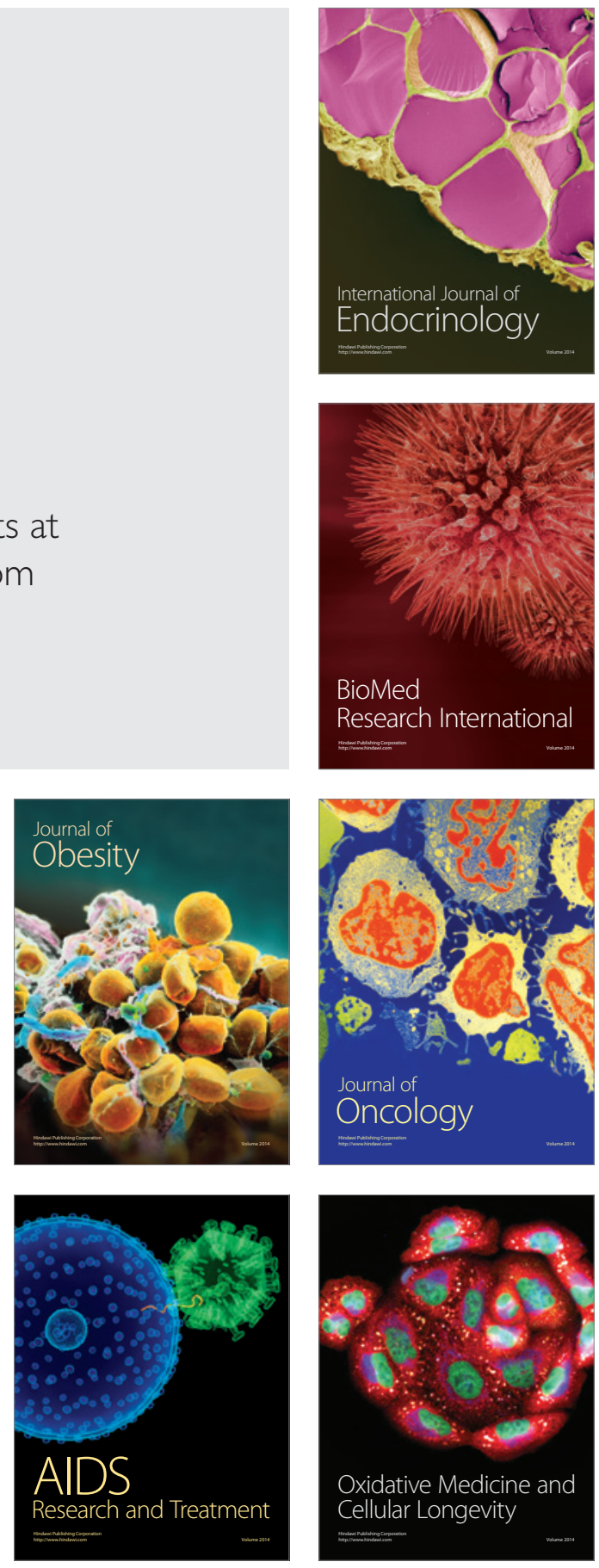